Original Research Paper

\title{
Computer Simulation of a Radiative MHD Flow in a Rotating Fluid
}

\author{
Harouna Naroua \\ Département de Mathématiques et Informatique, Université Abdou Moumouni, Niamey, Niger
}

Article history

Received: 08-09-2016

Revised: 31-10-2016

Accepted: 05-11-2016

Email: hnaroua@yahoo.com

\begin{abstract}
A hydromagnetic free convection flow has been modeled and simulated. The flow is considered unsteady, near a moving infinite flat plate in a rotating fluid. For the radiative heat transfer to be significant, very high temperatures are involved rendering the problem highly nonlinear. The simulation is based on a generic finite element scheme coupled with a stepwise Lagrange polynomial. The equations and other parameters entering into the description of the flow are transformed into interpretable postfix codes. Numerical values are computed for the temperature and velocity distributions. The results obtained are depicted graphically and discussed.
\end{abstract}

Keywords: Computer Simulation, Modeling, Generic Software, Numerical Scheme, Unsteady Flow

\section{Introduction}

The study of the magnetohydrodynamic flows is important because of its enormous applications in magnetohydrodynamic electrical power generation, geophysics, etc. As a result, considerable research efforts have been made by various authors such as Ali and Andallah (2016; Aiyesimi et al., 2016; Bachir and Naroua, 2015; Dhar et al., 2016; Ghosh and Pop, 2007; Medikare et al., 2016; Tokis, 1988; Tu, 2015; Yassen et al., 2016; Yusuf et al., 2016).

El-Dabe et al. (2015) used finite differences to investigate and numerically solve a magnetohydrodynamic micropolar flow problem. The investigated flow was in the presence of mass and heat transfer. They observed that their numerical solution is in good agreement with the analytical solutions. Ali et al. (2015) used the Homotopy Analysis Method to solve a fluid flow problem in a porous medium. For their analysis, they considered a viscous and incompressible fluid. The results obtained from their solution and the numerical results collected from the literature have been compared. Hossain et al. (2015) made use of the Galerkin finite element method to investigate a Magnetohydrodynamic problem. The temperature and velocity distributions are computed and analyzed.

Though the regularity of the problem is an important issue, we are proposing to solve a fluid flow problem with a generic computer tool using the Galerkin finite element scheme to compute the nodal values and generate the results from a stepwise Lagrange polynomial.

\section{Mathematical Formulation of the Problem}

The flow configuration and the equations governing the problem have been described by Naroua (2010). In non-dimensional form, the governing equations of the problem are:

$$
\begin{aligned}
& \left\{\frac{\partial u}{\partial t}-2 E v=\frac{\partial^{2} u}{\partial z^{2}}-M^{2} u+G r(\theta-1)\right. \\
& \left\{\frac{\partial v}{\partial t}+2 E u=\frac{\partial^{2} v}{\partial z^{2}}-M^{2} v\right. \\
& \left\{\operatorname{Pr} \frac{\partial \theta}{\partial t}=\frac{\partial^{2} \theta}{\partial z^{2}}-R\left(\theta^{4}-1\right)\right.
\end{aligned}
$$

with boundary conditions:

$$
\left\{\begin{array}{l}
u=1, \quad v=0, \quad \theta=\theta_{w} \quad \text { at } \quad z=0 \\
u \rightarrow 0, \quad v \rightarrow 0, \quad \theta \rightarrow 0 \quad \text { as } \quad z \rightarrow \infty
\end{array}\right.
$$

\footnotetext{
Where:

$\begin{array}{ll}u & =\text { The primary velocity } \\ v & =\text { The secondary velocity }\end{array}$
}




\section{$\theta \quad=$ The temperature \\ Gr, Pr, E, R and M = Respectively the Grashof number, the Prandtl number, the Eckert number, the radiation parameter and the magnetic parameter}

Using the proposed generic computer tool and the boundary conditions (4), we numerically solve the above system of Equations 1-3. At the elemental level:

$$
\begin{aligned}
& \phi_{1}^{(i)}(z)=\frac{\left(z_{i+1}-z\right)}{\left(z_{i+1}-z_{i}\right)} \\
& \phi_{2}^{(i)}(z)=\frac{\left(z-z_{i}\right)}{\left(z_{i+1}-z_{i}\right)}
\end{aligned}
$$

where, $1 \leq i \leq N, z_{i}$ represents the lower coordinate of the $i^{\text {th }}$ element, $z_{i+1}$ is the upper coordinate of the element $\mathrm{i}$, $\phi_{i}$ represents the test function and $\Omega$ represents the domain of the flow.

\section{Solution of the Energy Equation}

The first step is to solve Equation 3 by constructing its quasi-variational equivalent to obtain Equation 6:

$$
0=\int_{\Omega} \phi_{i}\left\{\operatorname{Pr} \frac{\partial \theta}{\partial t}-\frac{\partial^{2} \theta}{\partial z^{2}}+R\left(\theta^{4}-1\right)\right\} d z
$$

Next, we consider a Galerkin approximation of two parameters as developed and described by Reddy (1984):

$$
\theta(z, t)=\sum_{j=1}^{N} d_{j}^{1}(t) \phi_{j}(z)
$$

We then use Equation 5 and 7 to reduce Equation 6 to:

$$
\left[A^{1}\right]\left\{\frac{\partial d^{1}}{\partial t}\right\}+\left[B^{1}\right]\left\{d^{1}\right\}=\left\{P^{1}\right\}
$$

where:

$$
\left\{\begin{array}{l}
A_{i j}^{1}=\int_{\Omega} \operatorname{Pr} \phi_{i} \phi_{j} d z \\
B_{i j}^{1}=\int_{\Omega}\left(\frac{\partial \phi_{i}}{\partial z} \cdot \frac{\partial \phi_{j}}{\partial z}+R \phi_{i} \phi_{j} \theta^{3}\right) d z \\
P_{i}^{1}=\int_{\Omega} R \phi_{i} d z
\end{array}\right.
$$

Equation 8 can be rewritten with the help of the $\Theta$ operator to obtain (Reddy, 1984):

$$
\left[\hat{A}^{1}\right]\left\{d^{1}\right\}_{n+1}=\left[\hat{B}^{1}\right]\left\{d^{1}\right\}_{n}+\left\{\hat{P}^{1}\right\}
$$

where:

$$
\left\{\begin{array}{l}
{\left[\hat{A}^{1}\right]=\left[A^{1}\right]+\Theta \Delta t\left[B^{1}\right]} \\
{\left[\hat{B}^{1}\right]=\left[A^{1}\right]-(1-\Theta) \Delta t\left[B^{1}\right]} \\
{\left[\hat{P}^{1}\right]=\Delta t\left[\Theta\left\{P^{1}\right\}_{n+1}+(1-\Theta)\left\{P^{1}\right\}_{n}\right]}
\end{array}\right.
$$

Using the Galerkin method on a 64 elements mesh, we obtain the initial value $d^{1}$ given by:

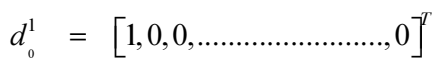

For $t>0$ :

$$
\left\{d^{1}\right\}_{n+1}=\left[\left(\hat{A}^{1}\right)\right]^{-1}\left[\hat{B}^{1}\right]\left\{d^{1}\right\}_{n}+\left[\left(\hat{A}^{1}\right)\right]^{-1}\left\{\hat{P}^{1}\right\}
$$

\section{Solutions of the momentum equations}

To compute these solutions, we need to solve Equations 1 and 2 by constructing their quasi-variational equivalent to obtain Equations 14 and 15:

$$
\begin{aligned}
& 0=\int_{\Omega} \phi_{i}\left\{\frac{\partial u}{\partial t}-2 E v-\frac{\partial^{2} u}{\partial z^{2}}+M^{2} u-G r(\theta-1)\right\} d z \\
& 0=\int_{\Omega} \phi_{i}\left\{\frac{\partial v}{\partial t}+2 E u-\frac{\partial^{2} v}{\partial z^{2}}+M^{2} v\right\} d z
\end{aligned}
$$

For each component of the velocity, we consider a Galerkin approximation of two parameters as developed and described by Reddy (1984):

$$
\begin{aligned}
& u(z, t)=\sum_{j=1}^{N} d_{j}^{2}(t) \phi_{j}(z) \\
& v(z, t)=\sum_{j=1}^{N} d_{j}^{3}(t) \phi_{j}(z)
\end{aligned}
$$

With the help of Equations 5, 16 and 17, Equations 14 and 15 can be rewritten as:

$\left[A^{2}\right]\left\{\frac{\partial d^{2}}{\partial t}\right\}+\left[B^{2}\right]\left\{d^{2}\right\}=\left\{P^{2}\right\}$

$$
\left[A^{3}\right]\left\{\frac{\partial d^{3}}{\partial t}\right\}+\left[B^{3}\right]\left\{d^{3}\right\}=\left\{P^{3}\right\}
$$


where:

$$
\left\{\begin{array}{l}
A_{i j}^{2}=\int_{\Omega} \phi_{i} \phi_{j} d z \\
B_{i j}^{2}=\int_{\Omega}\left(\frac{\partial \phi_{i}}{\partial z} \cdot \frac{\partial \phi_{j}}{\partial z}+M^{2} \phi_{i} \phi_{j}\right) d z \\
P_{i}^{2}=\int_{\Omega} \phi_{i}(2 E v+G r(\theta-1)) d z \\
A_{i j}^{3}=\int_{\Omega} \phi_{i} \phi_{j} d z \\
B_{i j}^{3}=\int_{\Omega}\left(\frac{\partial \phi_{i}}{\partial z} \cdot \frac{\partial \phi_{j}}{\partial z}+M^{2} \phi_{i} \phi_{j}\right) d z \\
P_{i}^{3}=-\int_{\Omega} 2 E \phi_{i} u d z
\end{array}\right.
$$

With the help of the $\Theta$-operator (Reddy, 1984), Equations 18 and 19 reduce to:

$$
\begin{aligned}
& {\left[\hat{A}^{2}\right]\left\{d^{2}\right\}_{n+1}=\left[\hat{B}^{2}\right]\left\{d^{2}\right\}_{n}+\left\{\hat{P}^{2}\right\}} \\
& {\left[\hat{A}^{3}\right]\left\{d^{3}\right\}_{n+1}=\left[\hat{B}^{3}\right]\left\{d^{3}\right\}_{n}+\left\{\hat{P}^{3}\right\}}
\end{aligned}
$$

where:

$$
\left\{\begin{array}{l}
{\left[\hat{A}^{2}\right]=\left[A^{2}\right]+\Theta \Delta t\left[B^{2}\right]} \\
{\left[\hat{B}^{2}\right]=\left[A^{2}\right]-(1-\Theta) \Delta t\left[B^{2}\right]} \\
{\left[\hat{P}^{2}\right]=\Delta t\left[\Theta\left\{P^{2}\right\}_{n+1}+(1-\Theta)\left\{P^{2}\right\}_{n}\right]} \\
{\left[\hat{A}^{3}\right]=\left[A^{3}\right]+\Theta \Delta t\left[B^{3}\right]} \\
{\left[\hat{B}^{3}\right]=\left[A^{3}\right]-(1-\Theta) \Delta t\left[B^{3}\right]} \\
{\left[\hat{P}^{3}\right]=\Delta t\left[\Theta\left\{P^{3}\right\}_{n+1}+(1-\Theta)\left\{P^{3}\right\}_{n}\right]}
\end{array}\right.
$$

Using the Galerkin method on a 64 elements mesh, we obtain the initial values $d_{0}^{2}$ and $d_{0}^{3}$ given by:

$$
\begin{aligned}
& d_{0}^{2}=[1,0,0, \ldots \ldots \ldots \ldots \ldots \ldots \ldots, 0]^{T} \\
& d_{0}^{3}=[0,0,0, \ldots \ldots \ldots \ldots \ldots \ldots \ldots . ., 0]^{T}
\end{aligned}
$$

For $t>0$ :

$$
\begin{aligned}
& \left\{d^{2}\right\}_{n+1}=\left[\left(\hat{A}^{2}\right)\right]^{-1}\left[\hat{B}^{2}\right]\left\{d^{2}\right\}_{n}+\left[\left(\hat{A}^{2}\right)\right]^{-1}\left\{\hat{P}^{2}\right\} \\
& \left\{d^{3}\right\}_{n+1}=\left[\left(\hat{A}^{3}\right)\right]^{-1}\left[\hat{B}^{3}\right]\left\{d^{3}\right\}_{n}+\left[\left(\hat{A}^{3}\right)\right]^{-1}\left\{\hat{P}^{3}\right\}
\end{aligned}
$$

Equations 13, 26 and 27 are used to compute the values of the temperature and velocity distributions at the nodes. The simulation results are computed from stepwise Lagrange polynomials that are constructed from the nodal values. All input elements are transformed into interpretable postfix codes which are used for computation purposes.

\section{Discussion of Results}

The numerical values for the flow fields (temperature and velocity) are calculated and examined both in the presence of cooling $(\mathrm{Gr}=+5)$ and heating $(\mathrm{Gr}=-5)$ of the plate by free convection currents. The results obtained are depicted graphically in Fig. 1-6 and are in agreement with Naroua (2010).

From Fig. 1, it is observed that:

- An increase in the Prandtl number (Pr) leads to a decrease in the temperature profile $(\Theta)$

- An increase in the radiation parameter $(\mathrm{R})$ leads to a fall in the temperature profile $(\Theta)$ whereas an increase in the time $(\mathrm{t})$ leads to a rise in the temperature profile $(\Theta)$

From Fig. 2-4, it is observed that:

- In both cases (cooling and heating of the plate), the primary velocity profile (u) changes insignificantly due to an increase in the radiation parameter $(\mathrm{R})$. It increases with an increase in the rotation parameter (E) and the time (t) whereas it falls with an increase in the Grashof number (Gr)

- An increase in the Prandtl number (Pr) leads to a fall in the primary velocity profile (u) in the first case (cooling of the plate); conversely, it leads to a rise in the primary velocity profile $(\mathrm{u})$ in the second case (heating of the plate)

- The primary velocity (u) is backward far away from the plate for larger positive $\mathrm{Gr}$

From Fig. 5 and 6, it is observed that:

- In the two cases (cooling and heating of the plate), the secondary velocity profile (v) changes insignificantly due to an increase in the radiation parameter $(\mathrm{R})$ whereas it falls with an increase in the rotation parameter $(E)$ and the time $(t)$

- The secondary velocity profile (v) increases in the first case (cooling of the plate) and decreases in the second case (heating of the plate) due to an increase in the Prandtl number (Pr) 


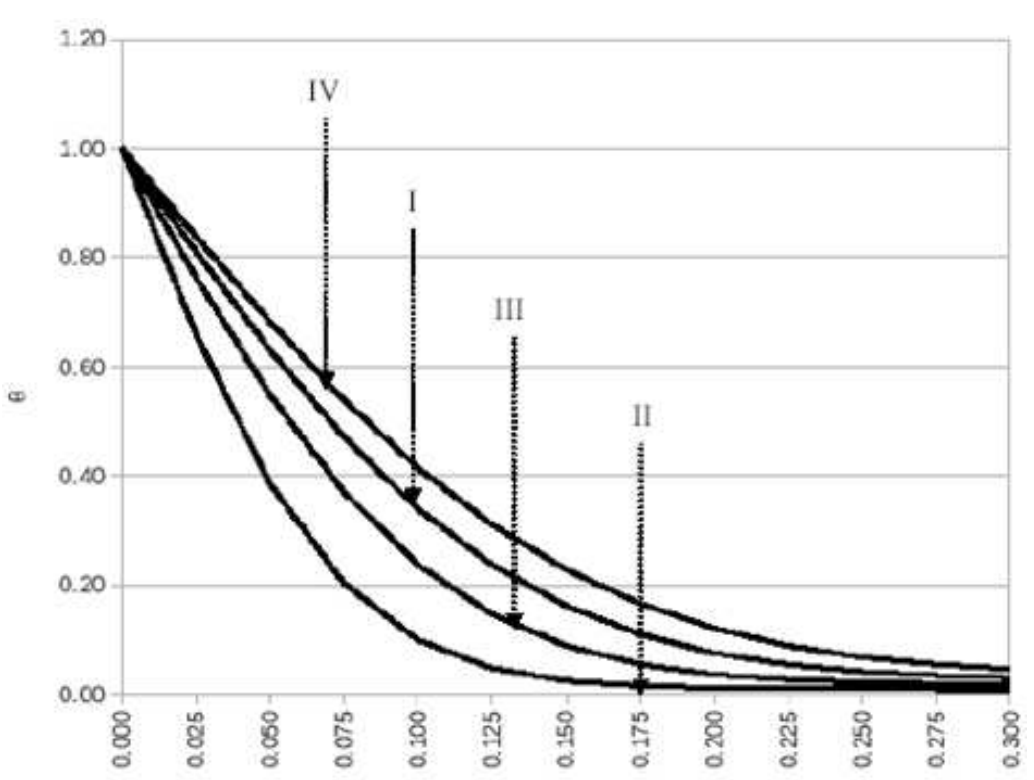

\begin{tabular}{|c|c|c|c|}
\hline \multicolumn{5}{|c|}{$z$} & R & $t$ \\
\hline Series & Pr & 5 & 0.001 \\
\hline I & 0.71 & 5 & 0.001 \\
\hline II & 7 & 10 & 0.001 \\
\hline IV & 0.71 & 5 & 0.005 \\
\hline
\end{tabular}

Fig. 1. Temperature distribution for $\mathrm{Gr}= \pm 5$

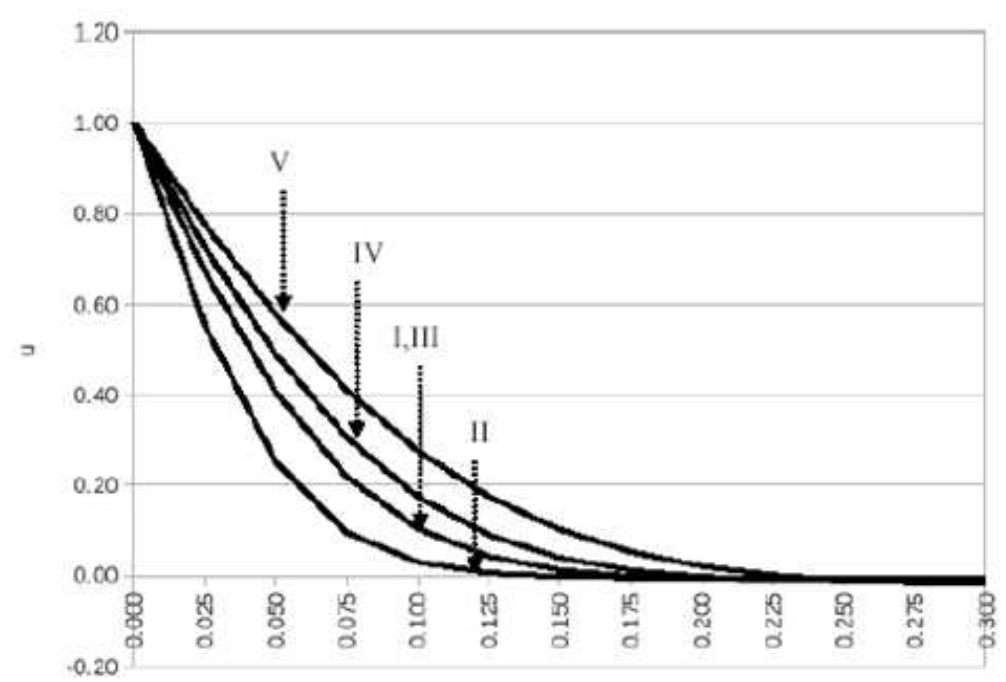

\begin{tabular}{|c|c|c|c|c|}
\hline Series & Pr & R & E & $t$ \\
\hline I & 0.71 & 5 & 0.1 & 0.001 \\
\hline II & 7 & 5 & 0.1 & 0.001 \\
\hline III & 0.71 & 10 & 0.1 & 0.001 \\
\hline IV & 0.71 & 5 & 0.5 & 0.001 \\
\hline V & 0.71 & 5 & 0.1 & 0.005 \\
\hline
\end{tabular}

Fig. 2. Primary velocity distribution for $\mathrm{Gr}=+5$ 


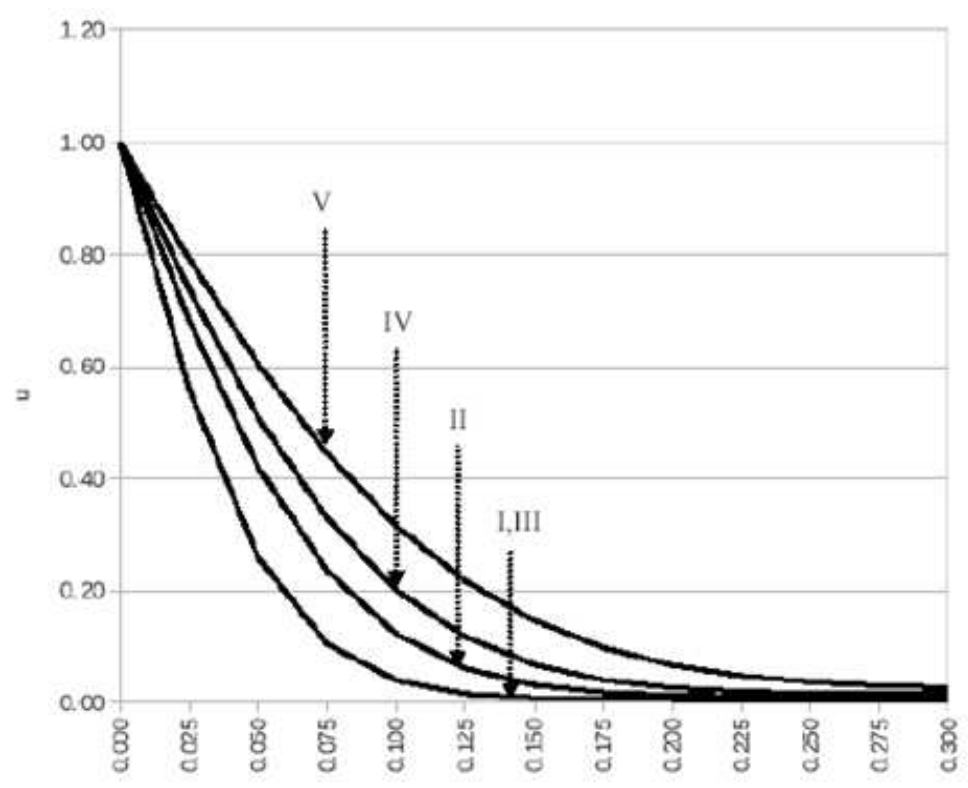

\begin{tabular}{|c|c|c|c|c|}
\hline Series & Pr & R & E & $t$ \\
\hline I & 0.71 & 5 & 0.1 & 0.001 \\
\hline II & 7 & 5 & 0.1 & 0.001 \\
\hline III & 0.71 & 10 & 0.1 & 0.001 \\
\hline IV & 0.71 & 5 & 0.5 & 0.001 \\
\hline V & 0.71 & 5 & 0.1 & 0.005 \\
\hline
\end{tabular}

Fig. 3. Primary velocity distribution for $\mathrm{Gr}=-5$

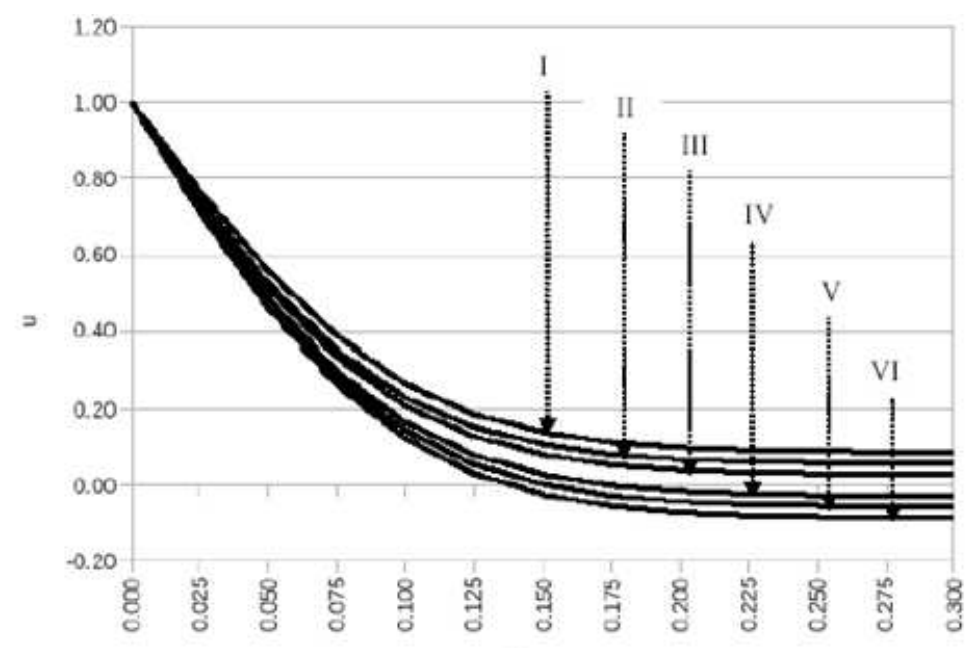

\begin{tabular}{|c|c|c|c|c|c|}
\hline Series & Gr & Pr & R & E & t \\
\hline I & -15 & 0.71 & 5 & 0.1 & 0.001 \\
\hline II & -10 & 0.71 & 5 & 0.1 & 0.001 \\
\hline III & -5 & 0.71 & 5 & 0.1 & 0.001 \\
\hline IV & +5 & 0.71 & 5 & 0.1 & 0.001 \\
\hline V & +10 & 0.71 & 5 & 0.1 & 0.001 \\
\hline VI & +15 & 0.71 & 5 & 0.1 & 0.001 \\
\hline
\end{tabular}

Fig. 4. Primary velocity distribution for Gr $\$ 0$ 


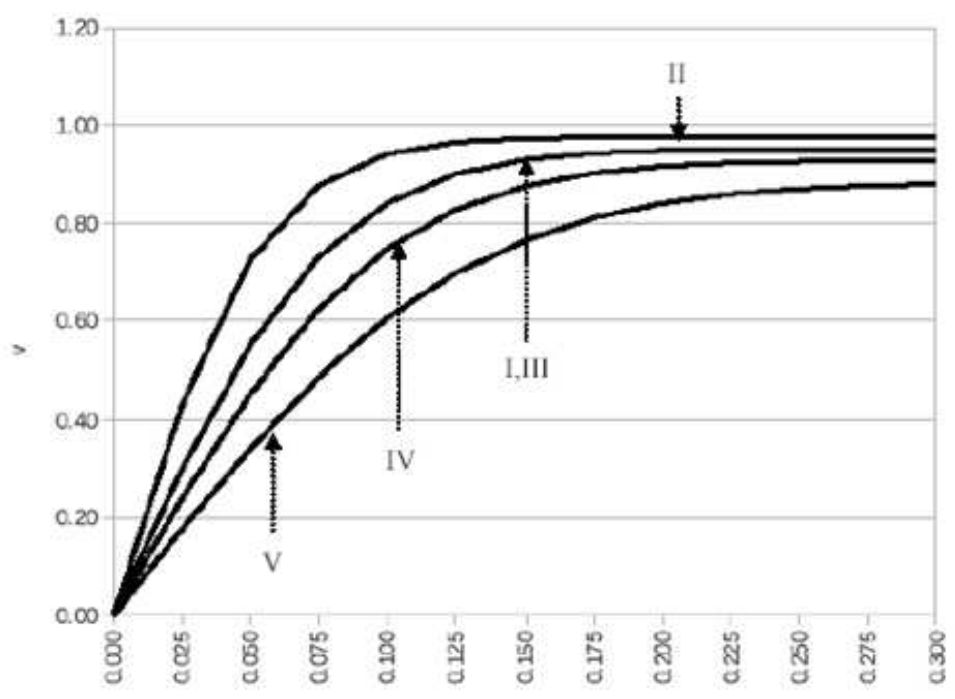

\begin{tabular}{|c|c|c|c|c|}
\hline Series & Pr & R & E & $t$ \\
\hline I & 0.71 & 5 & 0.1 & 0.001 \\
\hline II & 7 & 5 & 0.1 & 0.001 \\
\hline III & 0.71 & 10 & 0.1 & 0.001 \\
\hline IV & 0.71 & 5 & 0.5 & 0.001 \\
\hline V & 0.71 & 5 & 0.1 & 0.005 \\
\hline
\end{tabular}

Fig. 5. Secondary velocity distribution for $\mathrm{Gr}=+5$

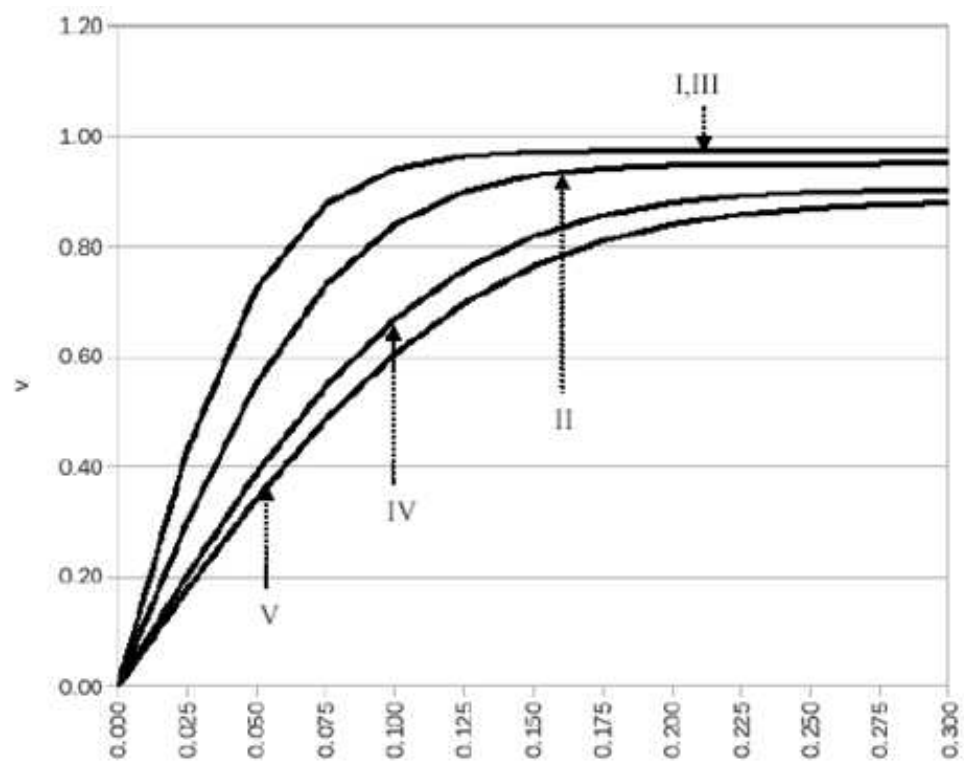

\begin{tabular}{|c|c|c|c|c|}
\hline Series & Pr & R & E & $t$ \\
\hline I & 0.71 & 5 & 0.1 & 0.001 \\
\hline II & 7 & 5 & 0.1 & 0.001 \\
\hline III & 0.71 & 10 & 0.1 & 0.001 \\
\hline IV & 0.71 & 5 & 0.5 & 0.001 \\
\hline V & 0.71 & 5 & 0.1 & 0.005 \\
\hline
\end{tabular}

Fig. 6. Secondary velocity distribution for $\mathrm{Gr}=-5$ 


\section{Conclusion}

In this article, a radiative MHD flow in a rotating fluid has been simulated. The simulation was based on a generic software tool using finite elements coupled with a stepwise Lagrange polynomial. From the results obtained, it is concluded that generic computer tools and interpretable codes can efficiently be used to solve fluid flow problems.

\section{Ethics}

This research paper is original and contains unpublished material. The author confirms that no ethical issues are expected to arise after its publication.

\section{References}

Aiyesimi, Y.M., M. Jiya, R.O. Olayiwola and A.A. Wachin, 2016. Mathematical analysis of convective flow of an unsteady Magnetohydrodynamic (MHD) third grade fluid in a cylindrical channel. Am. J. Comput. Applied Math., 6: $103-108$

Ali, A. and L. Andallah, 2016. Inflow outflow effect and shock wave analysis in a traffic flow simulation. Am. J. Comput. Math., 6: 55-65.

Ali, A., H. Zaman, M.Z. Abidin, Naeemullah and S.I.A. Shah, 2015. Analytic solution for fluid flow over an exponentially stretching porous sheet

with surface heat flux in porous medium by means of homotopy analysis method. Am. J. Comput. Math., 5: 224-238.

Bachir, M.I. and H. Naroua, 2015. A generic software tool for the simulation of laminar fluid flow problems. Eur. J. Scientific Res., 136: 292-302.

Dhar, D., M. Alim and L. Andallah, 2016. Group method analysis of MHD mixed convective flow past on a moving curved surface with suction. Am. J. Comput. Math., 6: 74-87.
El-Dabe, N.T., A.Y. Ghaly, R.R. Rizkallah, K.M. Ewis and A.S. Al-Bareda, 2015. Numerical solution of MHD flow of micropolar fluid with heat and mass transfer towards a stagnation point on a vertical plate. Am. J. Comput. Math., 5: 158-174.

Ghosh, S.K. and I. Pop, 2007. Thermal radiation of an optically thick gray gas in the presence of indirect natural convection. Int. J. Fluid Mechan. Res., 34: 515-520.

Hossain, S.A., M.A. Alim and S.K. Saha, 2015. A finite element analysis on MHD free convection flow in open square cavity containing heated circular cylinder. Am. J. Comput. Math., 5: 41-54.

Medikare, M., S. Joga and K. Chidem, 2016. MHD stagnation point flow of a casson fluid over a nonlinearly stretching sheet with viscous dissipation. Am. J. Comput. Math., 6: 37-48.

Naroua, H., 2010. Modeling of unsteady MHD free convection flow with radiative heat transfer in a rotating fluid. Nat. Sci., 2: 1384-1393.

Reddy, J.N., 1984. An Introduction to the Finite Element Method. Mc Graw Hill book company, Singapore.

Tokis, J.N., 1988. Free convection and mass transfer effects on the magnetohydrodynamic flows near a moving plate in a rotating medium. Astrophys. Space Sci., 144: 291-301.

Tu, S.Z., 2015. A riemann-solver free spacetime discontinuous galerkin method for general conservation laws. Am. J. Comput. Math., 5: 55-74.

Yassen, M., M. Sohaly and I. Elbaz, 2016. Random crank-nicolson scheme for random heat equation in mean square sense. Am. J. Comput. Math., 6: 66-73.

Yusuf, A., Y.M. Aiyesimi, M. Jiya and G.T. Okedayo, 2016. Analysis of couette flow of a nanofluid in an inclined channel with soret and dufour effects. Am. J. Comput. Applied Math., 6: 57-64. 